\title{
Meshless analysis of flow and concentration in a water reservoir
}

\author{
M. Kanoh ${ }^{1}$, N. Nakamura ${ }^{1}$, K. Kai ${ }^{1}$, T. Kuroki ${ }^{2}$ \& K. Sakamoto ${ }^{3}$ \\ ${ }^{I}$ Department of Civil Engineering, Kyushu Sangyo University, Japan \\ ${ }^{2}$ Baikoen 1-15-21 Dazaifu, Fukuoka 818-0124, Japan \\ ${ }^{3}$ Environment Division, Matsue Doken Co., Ltd. Japan
}

\begin{abstract}
In an earlier study, a boundary element methodology was developed to obtain numerically stable and convergent results for the concentration distribution and flow of a water reservoir. In the process to apply the boundary element method to the flow analysis around a machine that supplies dissolved oxygen (DO), we realised that the divergence and accuracy of the very delicate flow were sensitive to the mesh (domain) and boundary discretisation. In other words, it seemed difficult to determine the appropriate lengths of the mesh (domain) and boundary discretisation for obtaining stability and convergence in the computational analysis. In this paper, a new meshless method is presented, which overcomes the difficulties of the boundary element method described above. The method is based on the idea of mesh-free radial basis functions (RBFs), which is a collocation method. Referring to the velocity vectors of the water flow calculated by the weighted finite difference method (WFDM) and the finite element method (FEM) and observed in the model simulation of a water reservoir constructed in the sanitary and environmental engineering laboratory of Kyushu Sangyo University, the effect and accuracy of the alternative meshless method were estimated.
\end{abstract}

Keywords: flow and concentration in water reservoirs, meshless method, weighted finite difference method, finite element method, observed velocity in model simulation of water reservoir.

\section{Introduction}

The poor-oxygen layer, which is short of or lacking in dissolved oxygen (DO), sometimes results in pollution of the water in a reservoir. An attempt was made 
to ameliorate the concentration of oxygen in the lower layer of the reservoir by using a machine that supplies DO (Kanoh et al. [1]). In order to numerically confirm the efficiency of the improvement in DO, we applied a meshless method to represent and calculate the slow but very delicate flow caused by the DOsupplying machine. For this purpose, two techniques were used: (1) the first is the penalty method, in which the pressure terms are eliminated in the Navier-Stokes equations for the meshless method; (2) the second is to identify the boundary conditions for the velocity and concentration against the vertical wall or the bottom and on the free surface.

\section{Governing equations}

The three equations, which are continuous, Navier-Stokes (N-S) and convective-diffusion equations, govern the flow in the flow domains of a water reservoir. In the vertical $\left(\mathrm{x}_{1}, \mathrm{x}_{2}\right)$ plane, as illustrated in Figure 1, these equations are shown as follows:

$$
\begin{gathered}
\frac{\partial \mathrm{u}_{1}}{\partial \mathrm{x}_{1}}+\frac{\partial \mathrm{u}_{2}}{\partial \mathrm{x}_{2}}=0 \\
\frac{\partial u_{1}}{\partial t}+u_{1} \frac{\partial u_{1}}{\partial x_{1}}+u_{2} \frac{\partial u_{1}}{\partial x_{2}}-v\left(\frac{\partial^{2} u_{1}}{\partial x_{1}{ }^{2}}+\frac{\partial^{2} u_{1}}{\partial x_{2}{ }^{2}}\right)=-\frac{1}{\rho} \frac{\partial P}{\partial x_{1}} \\
\frac{\partial u_{2}}{\partial t}+u_{1} \frac{\partial u_{2}}{\partial x_{1}}+u_{2} \frac{\partial u_{2}}{\partial x_{2}}-v\left(\frac{\partial^{2} u_{2}}{\partial x_{1}{ }^{2}}+\frac{\partial^{2} u_{2}}{\partial x_{2}{ }^{2}}\right)=-\frac{1}{\rho} \frac{\partial P}{\partial x_{2}}+g \\
\frac{\partial T}{\partial t}+u_{1} \frac{\partial T}{\partial x_{1}}+u_{2} \frac{\partial T}{\partial x_{2}}-D\left(\frac{\partial^{2} T}{\partial x_{1}{ }^{2}}+\frac{\partial^{2} T}{\partial x_{2}{ }^{2}}\right)=0 \\
\rho=\mathrm{aT}+\mathrm{b}
\end{gathered}
$$

where $\mathrm{x}_{1}$ and $\mathrm{x}_{2}$ are the horizontal and vertical directions, $\mathrm{u}_{1}$ and $\mathrm{u}_{2}$ describe the velocities of the $x_{1}$ and $x_{2}$ directions, $P$ is the pressure, $g$ is the gravity acceleration, $v$ is the kinematic viscosity, $\mathrm{T}$ is the water temperature, and $\mathrm{D}$ is the diffusion coefficient. Here, the density $\rho$ is connected to the water temperature $T$, as written in Equation (4), with the coefficients a and b. The water temperature $\mathrm{T}$ is compatible to the concentration of dissolved oxygen (DO) in case it is necessary to calculate the DO or other values. 


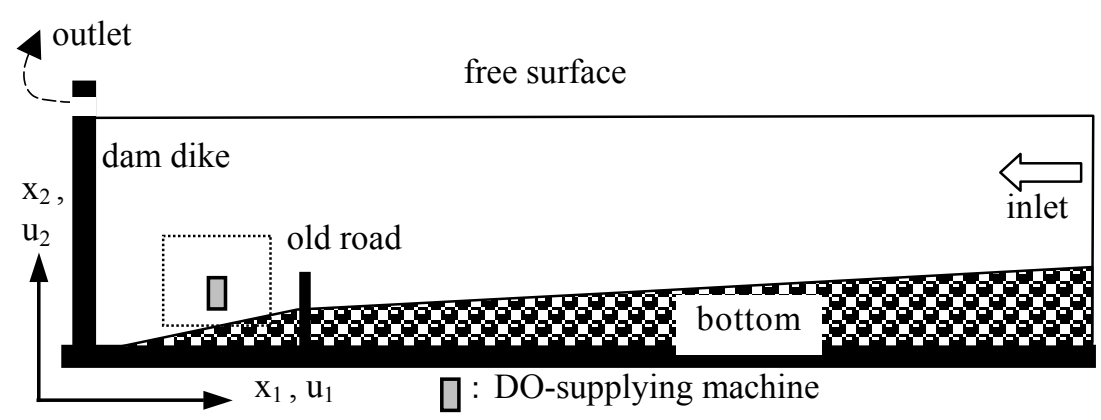

Figure 1: The entire domain of a water reservoir.

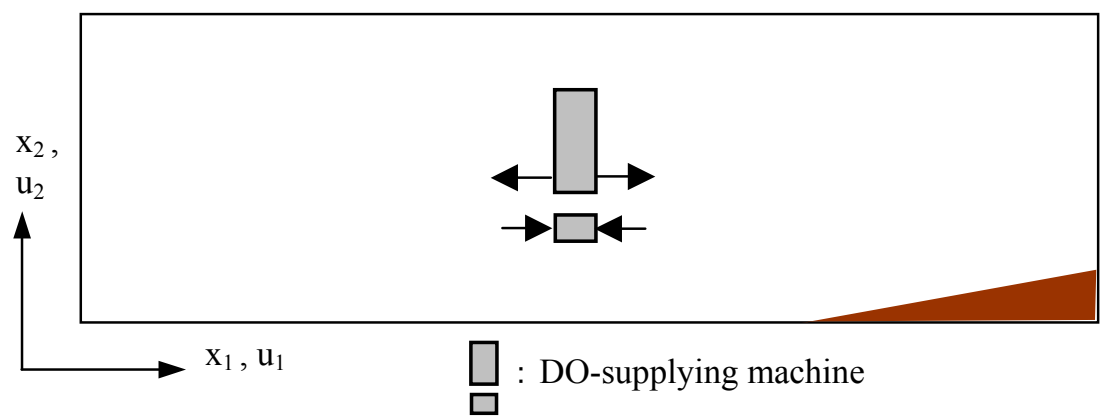

Figure 2: $\quad$ The domain around a DO-supplying machine in a reservoir.

\section{Application of the meshless method}

\subsection{Meshless method for flow and concentration analysis}

We deal with the flow and concentration analysis in the water reservoir as shown in Figures 1 and 2, and try to apply the meshless method to the problem. The meshless method is based on the idea of the mesh-free RBF collocation method (e.g., Divo et al. [2]). The penalty method was used so that the pressure terms would be eliminated in the $\mathrm{N}-\mathrm{S}$ equations and the difficulty of the pressure boundary conditions would be avoided in the meshless method. 


\subsection{Meshless method formulation}

\subsubsection{Application of the penalty method to $\mathrm{N}-\mathrm{S}$ equations}

In order to eliminate the pressure terms in the $\mathrm{N}-\mathrm{S}$ equations and avoid the difficulty of the pressure boundary conditions, the penalty method is introduced, and the following equations are obtained as described below

$$
u_{j} \cdot u_{i, j}-\lambda\left(u_{1,2}+u_{2,2}\right)_{, j} \cdot \delta_{i, j}-v\left(u_{1,2}+u_{2,2}\right)_{, j}=0 \quad \text { i, } \mathrm{j}=1,2
$$

where $\lambda$ means $R_{e} \cdot K / \rho, R_{e}$ is the Reynolds number, and $K$ describes the coefficient of the penalty method (Kanoh et al. [1]).

\subsubsection{Simultaneous equations for the meshless method}

Substituting $\underline{\mathrm{u}}_{1}$ and $\underline{\mathrm{u}}_{2}$ at time $(\mathrm{t}-\Delta \mathrm{t})$ into the above Equation (5), the following expression is obtained:

$$
\begin{aligned}
& \underline{\mathrm{u}}_{1} \cdot \mathrm{u}_{1,1}+\underline{\mathrm{u}}_{2} \cdot \mathrm{u}_{1,2}-\lambda\left(\mathrm{u}_{1,11}+\mathrm{u}_{2,21}\right)-\mathrm{v}\left(\mathrm{u}_{1,22}+\mathrm{u}_{2,12}+2 \mathrm{u}_{1,11}\right)=0 \\
& \underline{\mathrm{u}}_{1} \cdot \mathrm{u}_{2,1}+\underline{\mathrm{u}}_{2} \cdot \mathrm{u}_{2,2}-\lambda\left(\mathrm{u}_{1,12}+\mathrm{u}_{2,22}\right)-\mathrm{v}\left(\mathrm{u}_{2,11}+\mathrm{u}_{1,21}+2 \mathrm{u}_{2,22}\right)=0
\end{aligned}
$$

The global expansion function $\mathrm{Xj}\left(=\left(\mathrm{r}^{2}+\mathrm{c}^{2}\right)^{-1 / 2}\right)$ is employed here so that the unknowns $\left(\mathrm{u}_{1}, \mathrm{u}_{2}\right.$, and $\left.\mathrm{T}\right)$ at time $(\mathrm{t})$ can be developed for the mesh-free RBF collocation method, where $\mathrm{r}$ equals $\left\{\left(\mathrm{x}-\mathrm{x}_{\mathrm{j}}\right)+\left(\mathrm{y}-\mathrm{y}_{\mathrm{j}}\right)\right\}^{1 / 2}$ and $\mathrm{c}$ is the constant. The unknown values $\mathrm{u}_{1}, \mathrm{u}_{2}$, and $\mathrm{T}$ are expressed as Equation (7) using the global expansion function $\mathrm{Xj}$.

$$
\begin{gathered}
\mathrm{u}_{1}=\alpha_{\mathrm{j}} \mathrm{Xj}=\alpha_{\mathrm{j}} /\left(\mathrm{r}^{2}+\mathrm{c}^{2}\right)^{1 / 2} \\
\mathrm{u}_{2}=\beta_{\mathrm{j}} \mathrm{Xj}=\beta_{\mathrm{j}} /\left(\mathrm{r}^{2}+\mathrm{c}^{2}\right)^{1 / 2} \\
\mathrm{~T}=\gamma_{\mathrm{j}} \mathrm{Xj}=\gamma_{\mathrm{j}} /\left(\mathrm{r}^{2}+\mathrm{c}^{2}\right)^{1 / 2}
\end{gathered}
$$

The simultaneous equations for calculating the above unknowns $\left(\alpha_{j}, \beta_{j}\right.$, and $\left.\gamma_{j}\right)$ in the steady state are obtained as Equation (8).

$$
\begin{aligned}
& \left\{\left(\underline{u}_{1} \frac{\partial X_{j}}{\partial x_{1}}+\underline{u}_{2} \frac{\partial X_{j}}{\partial x_{2}}\right)-(v+\lambda) \frac{\partial^{2} X_{j}}{\partial x_{1}{ }^{2}}-v\left(\frac{\partial^{2} X_{j}}{\partial x_{1}{ }^{2}}+\frac{\partial^{2} X_{j}}{\partial x_{2}{ }^{2}}\right)\right\} \alpha_{j}-(v+\lambda) \frac{\partial^{2} X_{j}}{\partial x_{1} \partial x_{2}} \beta_{j}=0 \\
& -(v+\lambda) \frac{\partial^{2} X_{j}}{\partial x_{1} \partial x_{2}} \alpha_{j}+\left\{\left(\underline{u}_{1} \frac{\partial X_{j}}{\partial x_{1}}+\underline{u}_{2} \frac{\partial X_{j}}{\partial x_{2}}\right)-(v+\lambda) \frac{\partial^{2} X_{j}}{\partial x_{2}{ }^{2}}-v\left(\frac{\partial{ }^{2} X_{j}}{\partial x_{1}^{2}}+\frac{\partial^{2} X_{j}}{\partial x_{2}{ }^{2}}\right)\right\} \beta_{j}=0
\end{aligned}
$$




$$
\left\{\left(\underline{u}_{1} \frac{\partial X_{j}}{\partial x_{1}}+\underline{u}_{2} \frac{\partial X_{j}}{\partial x_{2}}\right)-D\left(\frac{\partial^{2} X_{j}}{\partial x_{1}^{2}}+\frac{\partial^{2} X_{j}}{\partial x_{2}^{2}}\right)\right\} \gamma_{j}=0
$$

Substituting the obtained values of $\alpha_{\mathrm{j}}, \beta_{\mathrm{j}}$, and $\gamma_{\mathrm{j}}$ into Equation (7), the values of three unknowns $\left(\mathrm{u}_{1}, \mathrm{u}_{2}\right.$, and $\left.\mathrm{T}\right)$ in the steady state can be calculated using the meshless method.

\subsection{Boundary conditions}

The boundary conditions for the free surface, the bottom and the vertical wall boundary have been previously proposed for BEM and WFDM in the flow region (Kanoh et al. [1]). Regarding the boundary conditions for flow analysis by our meshless method, the outline is as follows: (1) the velocities that exist in the normal and tangential directions at the wall or on the bottom are zero; (2) the pressure on the free surface is defined as zero. The pressure that is defined on the inside point neighbouring the wall or bottom is calculated as shown below:

$$
\mathrm{P}_{\text {inside }}=\mathrm{P}_{\text {outside }}-\rho \cdot v \cdot \mathrm{u}_{\mathrm{n}-\mathrm{l}} /(\Delta \mathrm{n}),
$$

where $\mathrm{P}_{\text {inside }}$ and $\mathrm{P}_{\text {outside }}$ are the pressures on the inside and outside points, respectively, and $\mathrm{u}_{\mathrm{n}-1}$ is the velocity value on the point that exists in the length of $\Delta \mathrm{n}$ to the boundary. This pressure boundary condition requires that the meshless method have the points existing inside the wall or bottom.

\section{Results and discussion}

The numerical results of the meshless method, the finite element method (FEM), and the weighted finite difference method (WFDM) are compared and discussed in this section. The two kinds of flow analysis calculated by these three methods are described here. Namely, the first analysis is the flow around a DO-supplying machine in a water reservoir, and the second is the flow analysis of an entire domain of the water reservoir.

\subsection{Flow analysis around a DO-supplying machine in a water reservoir}

As described above, in the process to apply the boundary element method to the flow analysis around the DO-supplying machine, we realised that the divergence and accuracy of the very delicate flow were sensitive to the mesh (domain) and boundary discretisation. In other words, it is difficult to determine the appropriate lengths of the mesh (domain) and boundary discretisation for obtaining convergence and stability in the computational analysis. The numerical results of the flow around the DO-supplying machine certified that our new meshless method overcame the difficulties of the boundary element method 


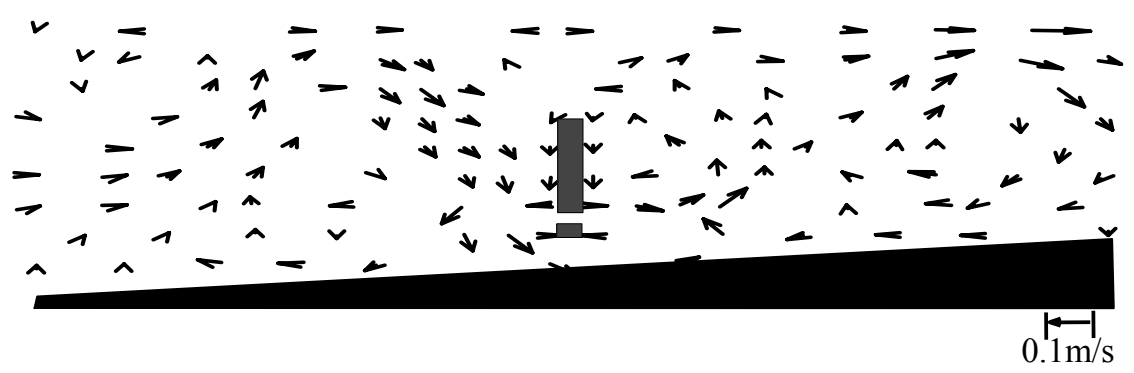

Figure 3: Velocity vectors using meshless method around a DO-supplying machine.

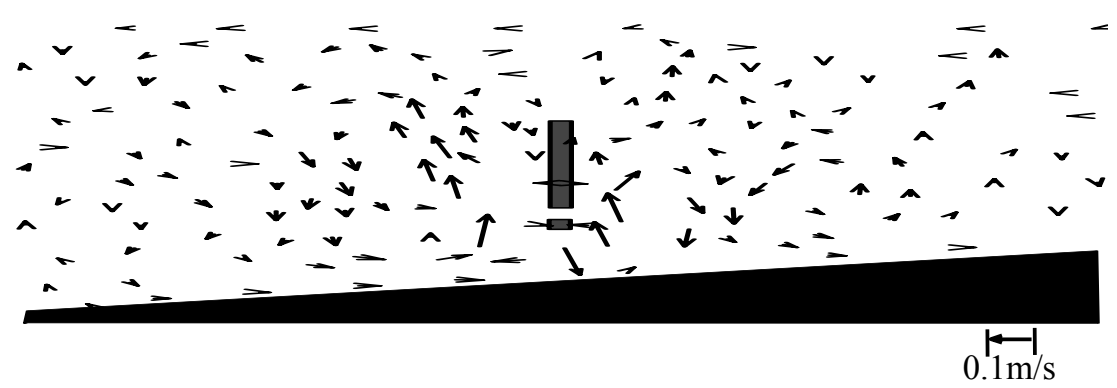

Figure 4: Velocity vectors using FEM around a DO-supplying machine.

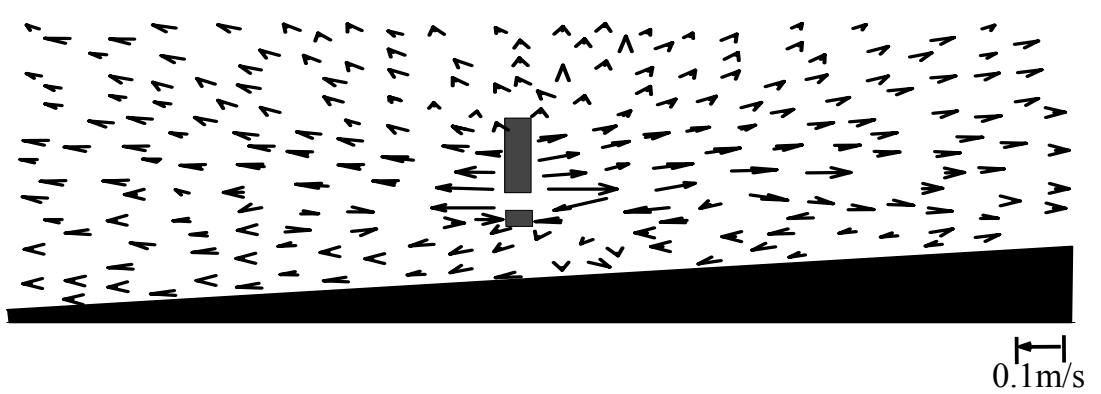

Figure 5: Velocity vectors using WFDM around a DO-supplying machine.

described above. It was reported in our previous work that the poor oxygen concentration frequently yielded a bad odour and dissolved heavy metals in the lower water layer of the B water reservoir. We were successful at improving the poor oxygen concentration in the lower layer of the B water reservoir using the DO-supplying machine. In order to economically improve the poor oxygen concentration of other water reservoirs, it seemed necessary to appropriately determine the capacity and the number of the DO-supplying machines required for improving the poor oxygen. For that purpose, we calculated the flow around a DO-supplying machine in a water reservoir by using the three methods described in this paper. 


\subsubsection{Meshless method calculation of the flow around the machine}

Figure 3 illustrates the flow velocity vector distribution around a DO-supplying machine in a water reservoir calculated by the meshless method, in which the number of the points in the meshless method is 651 and the three values of $\lambda, C$, and $v$ are $1000.0,1.0$, and 0.001 , respectively. The boundary element method could not yield stable and convergent results to the flow analysis around the machine to supply DO; on the other hand, the stability and convergence of the flow analysis using the meshless method seemed satisfactory.

\subsubsection{FEM calculation of the flow around the machine}

Figure 4 illustrates the flow velocity vector distribution around a DO-supplying machine in a water reservoir calculated by the finite element method, in which the number of the elements in the FEM is 300 and the two values of $\lambda$ and $v$ are 1000.0 and 0.001 , respectively. The penalty method was also introduced so that the pressure terms would be eliminated in the Navier-Stokes equations and the difficulty of the pressure boundary conditions could be avoided in the finite element method as well as in the meshless method. The kinds of flow velocity vector distributions around the DO-supplying machine calculated by both the finite element method and the meshless method closely resemble each other. The stability and convergence of the flow analysis using the finite element method also seemed satisfactory.

\subsubsection{WFDM calculation of the flow around the machine}

Figure 5 illustrates the flow velocity vector distribution around a DO-supplying machine in a water reservoir calculated by the weighted finite difference method, in which the number of meshes in the WFDM is 4750 and the value of $v$ is 0.0000015 . We consider that the flow velocity vectors around the DO-supplying machine calculated by the WFDM are reasonable, since the WFDM yielded very similar solutions to the true results of several flow problems that were observed in the simulation models constructed in the sanitary and environmental engineering laboratory of Kyushu Sangyo University.

\subsubsection{Domain discretisation of the four methods around a DO-supplying machine}

Figure 6 shows the domain discretisation of the four methods around a DOsupplying machine. Comparing the node distribution of the meshless method with the boundary element distribution of the BEM around the DO-supplying machine, we can see that both the number of the points of the meshless method and the number of the boundary elements of the BEM closely resemble each other. As described above, the BEM could not give stability to the flow analysis around the DO-supplying machine; on the other hand, the stability of our meshless method was satisfactory. Namely, our meshless method overcame the difficulty of the BEM to determine the appropriate combinations of the lengths of the domain and boundary discretisation for obtaining the stability in the computational analysis. Comparing the node distribution of the meshless method 
with those of the FEM and WFDM in Figure 6(a), (c), and (d), we can see that the three sets of the number of the points of the meshless method, the finite elements of the FEM, and the meshes of the WFDM are almost identical. Moreover, all three methods can give stability and convergence to the flow analysis around the DO-supplying machine.

(a) Node distribution of the meshless method around a DO-supplying machine.

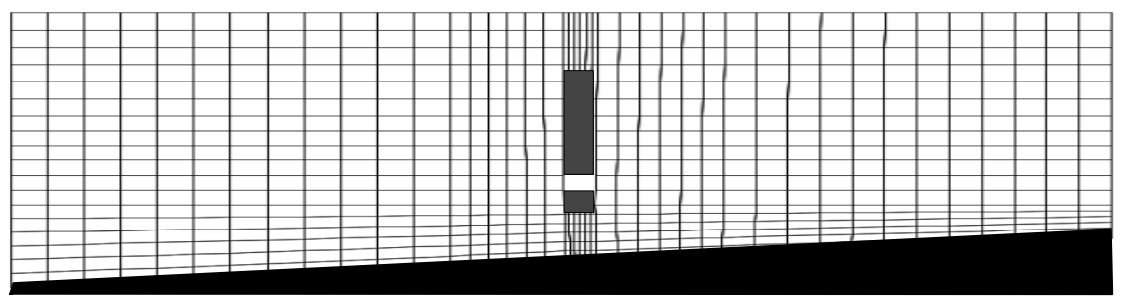

(b) Boundary element distribution of BEM around a DO-supplying machine.

(c) Element distribution of FEM around a DO-supplying machine.

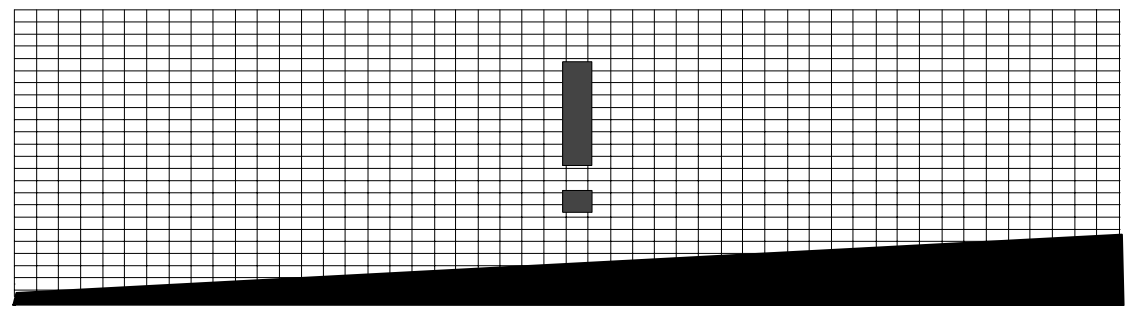

(d) Mesh distribution of WFDM around a DO-supplying machine.

Figure 6: Domain discretisation of the four methods around a DO-supplying machine. 


\subsection{Flow analysis of the entire domain of a water reservoir}

\subsubsection{Meshless method calculation of the entire domain}

Figure 7 illustrates the velocity vectors of the entire domain in the B water reservoir calculated using the meshless method, in which the number of the points in the meshless method is 801 and the three values of $\lambda, C$, and $v$ are 1000.0, 0.1, and 0.001, respectively. We consider that the stability and convergence of the meshless method for this problem are satisfactory.

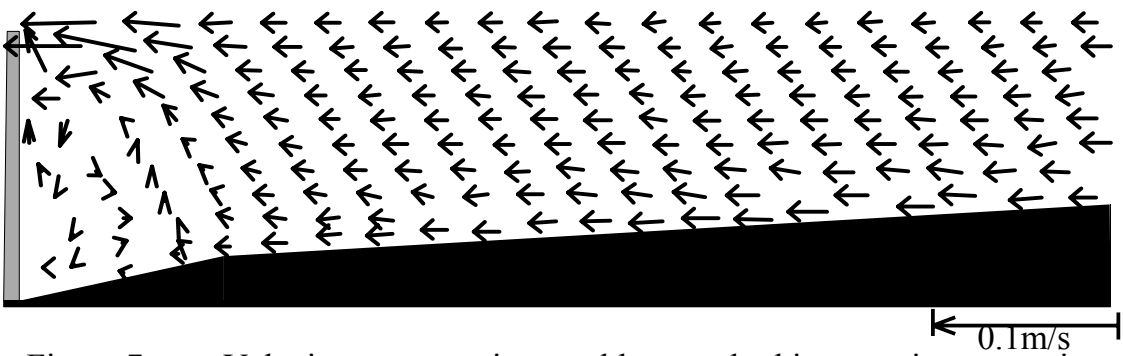

Figure 7: Velocity vectors using meshless method in an entire reservoir.

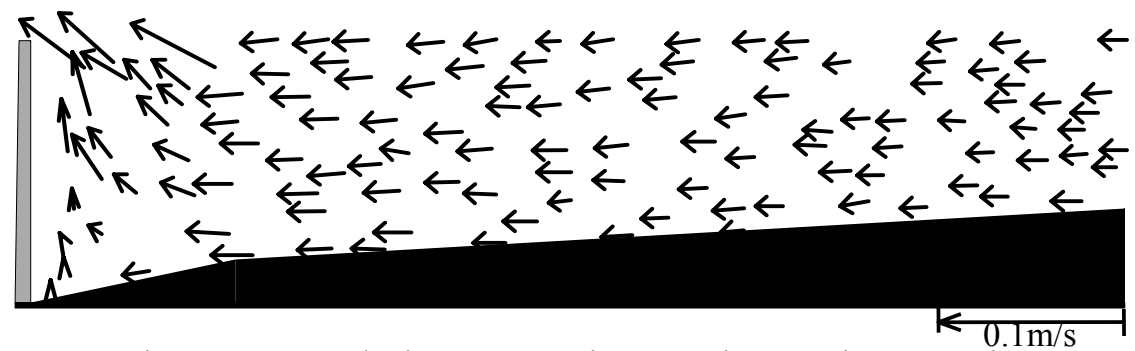

Figure 8: Velocity vectors using FEM in an entire reservoir.

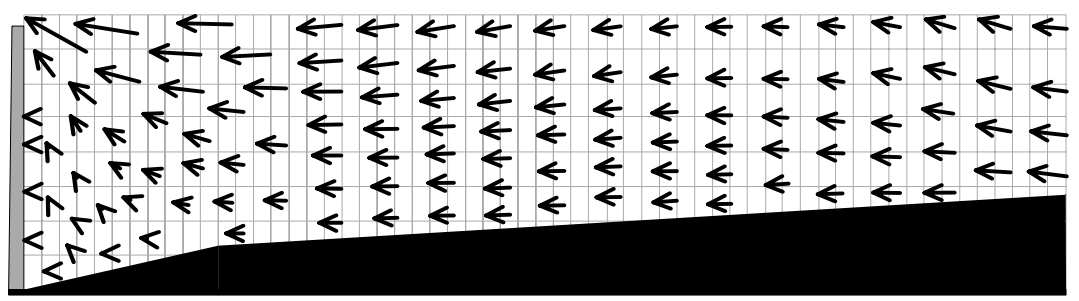

Figure 9: Velocity vectors using WFDM in an entire reservoir.

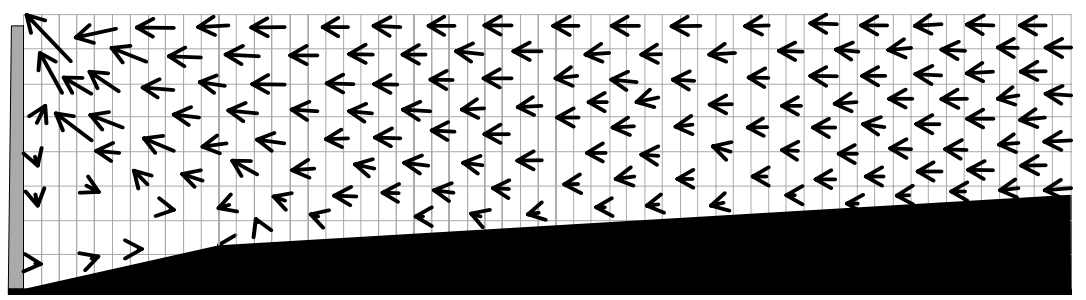

Figure 10: Observed velocity vectors in a model simulation of a reservoir. 


\subsubsection{Finite element method calculation of the whole domain}

Figure 8 shows the velocity vectors of the entire domain in the B water reservoir calculated using the finite element method, in which the number of the elements in the FEM is 800 and the two values of $\lambda$ and $v$ are 1000.0 and 0.001 , respectively. We consider that the stability and convergence of the FEM for this problem are also satisfactory.

\subsubsection{Weighted finite difference method calculation of the whole domain}

Figure 9 illustrates the velocity vectors of the entire domain in the B water reservoir calculated using the weighted finite difference method, in which the number of meshes in the WFDM is 2905 and the value of $v$ is 0.000001 . We consider that the divergence and accuracy of the WFDM for this problem are satisfactory enough. Figure 10 illustrates the velocity vectors of the entire domain in the B water reservoir observed in the hydro-model constructed in the sanitary and environmental engineering laboratory of Kyushu Sangyo University. Comparing the observed values with the calculated results using the WFDM, we could see that the WFDM could yield a very similar solution to the actual results of the problem.

\section{Conclusion}

In order to overcome the fact that the boundary element method could not give stability and convergence to the flow analysis around the DO-supplying machine, we applied a meshless method to analyse the problem. The meshless method could calculate two kinds of flow; the first is that caused by the DOsupplying machine, and the second is the flow distribution of the entire domain in the B water. The stability and convergence of the two kinds of flow analysis using the meshless method seemed satisfactory. Comparing the observed values with the calculated results using the WFDM, we can see that the WFDM can yield a very similar solution to the actual results of the flow analysis of the entire domain in the B water reservoir. By investigating the methodology, the boundary conditions, and other techniques of the WFDM, we intend to develop a meshless method for the flow and concentration analysis in a water reservoir.

\section{References}

[1] Kanoh, M., Nakamura, N., and Kuroki T., Boundary element method for the analysis of flow and concentration in a water reservoir, Proc. of the $28^{\text {th }}$ World Conf. on Boundary Elements and Other Mesh Reduction Methods, ed. C.A. Brebbia, WIT Press, Skiathos, Greece, pp. 231-240, 2006.

[2] Divo E., Kassab A., and Zahab El., Parallel domain decomposition meshless modeling of dilute convection-diffusion of species, Proc. of $27^{\text {th }}$ World Conf. on Boundary Elements and Other Mesh Reduction Methods, ed. C.A. Brebbia, WIT Press, Florida, USA, pp.79-89, 2005. 